\title{
Left ventricular systolic and diastolic function in children with overweight and obesity
}

\author{
Schusterova $\mathrm{I}^{1}$, Jurko $\mathrm{A}^{2}$, Minarik $\mathrm{M}^{3}$ \\ Eastern Slovakian Institute of Cardiovascular Diseases, Kosice, Slovakia. ajurko@zmail.sk
}

\begin{abstract}
Objectives: Several studies have found an association between obesity and heart failure. The purpose of our study was to identify the influence of the severity of obesity in children on structural and functional changes of the left ventricle and parameters of the systolic and diastolic function.

Methods: This was a prospective controlled study carried out between February 1 and October 31, 2010, involving 21 children with overweight and obesity and 23 healthy controls. Following parameters were evaluated: nutritional status, intermittent and continuous ambulatory blood pressure monitoring, echocardiographic examination including aortal root diameter, left atrial diameter, interventricular septum thickness, left ventricular end-diastolic diameter, left ventricular end-systolic diameter, left ventricular posterior wall thickness, left ventricle mass, relative wall thickness, left ventricular mass/height index and ejection fraction.

Results: In overweight and obese children, systolic blood pressure, diastolic blood pressure, average day-time SBP were higher than in controls. Also, left ventricular size was increased and diastolic dysfunction with preserved systolic function was present in overweight children.

Conclusions: In our study we have found that in obese and overweight children signs of early myocardial damage, both structural and functional, are detectable mainly as the alteration of the left ventricular diastolic function, despite preserved global systolic function. These changes seem to be intensified by the severity of obesity (Tab. 3, Ref. 39). Full Text in PDF www.elis.sk.

Key words: children, obesity, myocardial function, hypertension, echocardiography.
\end{abstract}

Recently, extreme increase of obesity has been reported not only in adults, but also in children. In the European Union similar trends have been observed for the last 20 years, with more significant increase of obesity in recent 10 years (1). Many studies have proven the relation between obesity and heart failure (2). In obese individuals, a wide spectrum of cardiovascular changes can be observed, starting from hyperdynamic circulation up to the changes of the myocardial structure (3). Similarly to adult patients, changes in left ventricular diastolic function indexes occur also in obese children and adolescents (4).

The purpose of our study was to identify the influence of the severity of obesity in children on structural and functional changes of the left ventricle and parameters of the systolic and diastolic function in pediatric patients with obesity and overweight.

\section{Methods}

The study was done at the Department of Pediatrics, Children's Faculty Hospital in Kosice, Slovakia between February 1 and October 31,2010. Study population consisted of two groups, the first

${ }^{1}$ Eastern Slovakian Institute of Cardiovascular Diseases, Kosice, Slovakia, ${ }^{2}$ Pediatric Cardiology Clinic, Martin, Slovakia, and ${ }^{3}$ Catholic University in Ružomberok, Faculty of Health Care, Ruzomberok, Slovakia

Address for correspondence: A. Jurko, Jr, MD, PhD, Department of Pediatrics, Pediatric Cardiology Clinic, Kollarova 13, SK-036 01, Martin, Slovakia.

Phone: +421.905324039 , Fax: +421.43 .7233010$ group included 21 children with overweight and obesity (13 with overweight and 8 with obesity), with average age of $13.5 \pm 1.2$ years. The second control group included 23 healthy children of similar age. The study was approved by the institutional ethics committee.

Nutritional status was evaluated by using body mass index (BMI), calculated as weight divided by square of height in meters. BMI percentile was identified from standard population charts for age and gender. BMI $<85$ th percentile was considered as normal body mass, BMI between 85 th and 95 th percentile as overweight and BMI above 95 th percentile as obesity (5).

Blood pressure was measured during clinic visit using a mercury pressure gauge following recommendations of the American Cardiologic Association (6). Mean systolic and diastolic blood pressures were used for statistical analysis. Ambulatory 24-blood pressure hours monitoring (AMBP) was carried out by using the oscillometric device Ergoscan with appropriate cuff size in accordance with the nondominant upper limb shoulder circumference. Only examinations with at least 5 successful night measurements and at least 15 measurements during the day were statistically processed. Blood pressure was measured each 15 minutes in the day-time (8.00-22.00 hours) and once an hour at night (22.00-8.00 hours). When processing AMBP data, the following parameters have been used: average day-time, night-time and 24-hour systolic and diastolic blood pressure. Belt diameter was measured at umbilical level.

Echocardiographic examination was done with Vivid 5 echocardiograph, using the $3.5 \mathrm{MHz}$ probe $\mathrm{S} 611$, always by the same cardiologist. The echocardiographic examination included a com- 
prehensive 2-D examination, colour and spectral Doppler examination and complete examination in M-mode acquired from 2-D projection. The techniques used to measure the left ventricle (LV) inner diameter, interventricular septal thickness and LV posterior wall thickness measured in diastole comply with the recommendations of the American Society of Echocardiography (7). The following parameters have been evaluated: aortal root diameter (Ao), left atrial diameter (LAD), interventricular septum thickness (IVS), left ventricular end-diastolic diameter (LVEDD), left ventricular end-systolic diameter (LVESD) and left ventricular posterior wall thickness (PWTh), left ventricle mass (LVM), calculated according to the Devereux's formula and the Penn convention (LVM $(\mathrm{g})=$ $1.04\left[(\text { LVEDD + IVST + LVPWT })^{3}-\right.$ LVEDD $\left.^{3}\right]-13.6$ g), relative wall thickness (RWTh), calculated from equation: RWTh $=$ IVST + LVPWT/LVEDD, left ventricular mass/height index was calculated as: $\operatorname{LVMIV}=\operatorname{LVM}(\mathrm{g}) /$ height $\left(\mathrm{m}^{2.7}\right)(8,9,10)$. Ejection fraction $(\mathrm{EF})$ was calculated according to the formula: EF $(\%)=$ LVEDV-LVESV/ LVEDV x $100 \%$, where LVEDV is the left ventricular end-diastolic volume and LVESV is the left ventricular end-systolic volume [13]. Stroke volume (SV) was calculated as a difference between the LVEDV and the LVESV. The recommendations of the American Society of Echocardiography were used to determine the diastolic function and its individual indexes (11). The pulse Doppler examination of flow through the mitral valve was used to obtain and evaluate the following parameters: E-wave (peak velocity of early diastolic filling), A-wave (peak velocity of late diastolic filling), E/A wave ratio, deceleration time of early diastolic filling (DT) and isovolumic relaxation time (IVRT).

Obtained data were processed by using the methods of descriptive and inductive statistics depending on the type and number of variables. For the purpose of the inductive statistics we assumed, that our data represent a random sample of the relevant population. The significance level was set to $5 \%$. The first step was a one- dimensional analysis - the tabulation of all monitored variables using frequency tables. All identified problems were checked and corrected. The second step was a two-dimensional analysis - the assessment of pairs of monitored variables. The nonparametric Kendall correlation coefficient (and the test of its significance), which measures the monotone statistical dependence (not only linear), was used for two numeric variables. It has been chosen due to the fact, that some variables had significantly abnormal distribution often with extreme values, which could eventually disvalue the traditionally used Pearson correlation coefficient. In order to compare numerical and categorical variables, description tables and the "eta" coefficient were used. ANOVA was used to determine the statistical significance of the mean difference. The distribution of variables was about normal. The last step was a multi-dimensional analysis - a multiple regression, where the relation between several numerical variables was examined simultaneously. All calculations were performed using a freeware version of SPSS.

\section{Results}

Systolic and diastolic blood pressure taken during clinic visit and average daily systolic blood pressure have been significantly higher in obese and overweight children. In children with obesity, there was also significantly higher average day-time systolic blood pressure. Interventricular septum, LV end diastolic diameter, LV end systolic diameter and also LV posterior wall thickness have been significantly larger in obese and overweight children. Left ventricular mass and also LV mass-to-height index have been significantly higher in overweight children (Tab. 1).

Parameters of left ventricular function, as LV end diastolic volume, LV end systolic volume, LV stroke volume have been significantly increased in overweight children except for LV ejec-

Tab. 1. Blood pressure and left ventricular dimensions.

\begin{tabular}{|c|c|c|c|c|c|c|}
\hline & \multicolumn{2}{|c|}{ Normal body weight } & \multicolumn{2}{|c|}{ Overweight } & \multicolumn{2}{|c|}{ Obesity } \\
\hline & Mean & $\mathrm{SD}$ & Mean & SD & Mean & $\mathrm{SD}$ \\
\hline$\overline{\text { Age (years) }}$ & 13.5 & 1.1 & 13.5 & 1.3 & 13.5 & 1.3 \\
\hline Body weight (kg) & 44.3 & 12.2 & $71.7 *$ & 15.1 & $77.4^{*}$ & 14.1 \\
\hline Height $(\mathrm{cm})$ & 151.9 & 23.2 & 165.3 & 10.0 & 166.0 & 9.1 \\
\hline $\mathrm{SBP}(\mathrm{mm} \mathrm{Hg})$ & 106.2 & 11.2 & $116.1 * * *$ & 10.3 & $120.0 * *$ & 8.5 \\
\hline DBP (mm Hg) & 65.6 & 5.9 & $71.6 * *$ & 8.0 & $73.0 * * *$ & 8.6 \\
\hline Average 24-hours SBP & 108.7 & 6.1 & 112.7 & 6.3 & $115.0 *$ & 6.4 \\
\hline Average day-time SBP & 113.06 & 6.0 & $119.4 * *$ & 8.1 & $122.8 * * *$ & 8.6 \\
\hline Average 24-hours DBP & 59.8 & 5.1 & 61.7 & 4.1 & 62.4 & 5.7 \\
\hline Average day-time DBP & 64.0 & 6.6 & 67.5 & 6.6 & 68.6 & 8.1 \\
\hline Average night-time SBP & 100.8 & 9.3 & 105.7 & 11.4 & 108.9 & 11.5 \\
\hline Average night-time DBP & 51.6 & 6.5 & 52.3 & 6.3 & 53.3 & 7.0 \\
\hline IVS (mm) & 7.42 & 1.32 & $8.53 *$ & 1.37 & $8.84 *$ & 1.53 \\
\hline LVEDD (mm) & 42.47 & 4.44 & $46.61 * * *$ & 4.0 & $47.45 * * * *$ & 3.30 \\
\hline $\operatorname{LVESD}(\mathrm{mm})$ & 28.21 & 3.43 & $31.32 * * *$ & 3.32 & $31.83 * * *$ & 3.51 \\
\hline RWTh & 0.33 & 0.04 & 0.35 & 0.04 & 0.35 & 0.03 \\
\hline LVM (g) & 104.14 & 36.88 & $149.55 * * * *$ & 46.1 & $152.59 * * * *$ & 43.65 \\
\hline LVMIV (g/m2.7) & 30.08 & 9.2 & $37.13 * *$ & 9.24 & $38.38 * *$ & 10.79 \\
\hline
\end{tabular}

SD - standard deviation, SBP - systolic blood pressure, DBP - diastolic blood pressure, IVS - interventricular septum thickness, LVEDD - left ventricular end-diastolic diameter, LVESD - left ventricular end-systolic diameter, PWTh - posterior wall thickness, RWTh - relative wall thickness, LVM - left ventricular mass, LVMIV - left ventricular mass index to height 2,7

$* \mathrm{p}<0.01, * * \mathrm{p}<0.001, * * * \mathrm{p}<0.005, * * * * \mathrm{p}<0.001$ 
Tab. 2. Parameters of left ventricular systolic and diastolic function.

\begin{tabular}{|c|c|c|c|c|c|c|}
\hline & \multicolumn{2}{|c|}{ Normal body weight } & \multicolumn{2}{|c|}{ Overweight } & \multicolumn{2}{|c|}{ Obesity } \\
\hline & Mean & SD & Mean & $\mathrm{SD}$ & Mean & $\mathrm{SD}$ \\
\hline LVEDV (ml) & 75.08 & 19.08 & $95.05 *$ & 20.1 & $100.03 * * *$ & 17.46 \\
\hline LVESV (ml) & 28.66 & 8.81 & $37.33 * *$ & $10 . .46$ & $37.71 * *$ & 10.43 \\
\hline $\mathrm{SV}(\mathrm{ml})$ & 46.2 & 10.94 & $58.41 * *$ & 16.5 & $61.91 * *$ & 16.37 \\
\hline $\mathrm{EF}(\%)$ & 61.92 & 3.52 & 60.72 & 8.7 & 61.97 & 9.8 \\
\hline LAD (mm) & 30.68 & 5.12 & $33.8 *$ & 3.69 & $34.38 *$ & 3.47 \\
\hline Ao/LA ratio & 1.29 & 0.18 & 1.33 & 0.12 & 1.34 & 0.11 \\
\hline A wave $(\mathrm{m} / \mathrm{s})$ & 0.48 & 0.12 & $0.59 *$ & 0.14 & $0.63 * *$ & 0.15 \\
\hline E wave $(\mathrm{m} / \mathrm{s})$ & 1.00 & 0.13 & 1.01 & 0.12 & 1.01 & 0.11 \\
\hline $\mathrm{E} / \mathrm{A}$ ratio & 2.19 & 0.65 & $1.80 *$ & 0.45 & $1.68^{*}$ & 0.42 \\
\hline DT (ms) & 150.45 & 40.36 & 169.0 & 44.25 & $180.36^{*}$ & 41.47 \\
\hline IVRT (ms) & 101.78 & 15.71 & 112.47 & 19.36 & $115.9 *$ & 19.27 \\
\hline
\end{tabular}

SD - standard deviation, LVEDV - left ventricular end-diastolic volume, LVESV - left ventricular end-systolic volume, SV - stroke volume, EF - ejection fraction, LAD left atrium diameter, Ao - aortic root size, A wave - peak velocity of late diastolic filling, E wave - peak velocity of early diastolic filling, DT - deceleration time of early transmitral blood flow velocity, IVRT - isovolumetric relaxation time

$* \mathrm{p}<0.01, * * \mathrm{p}<0.001, * * * \mathrm{p}<0.005, * * * * \mathrm{p}<0.0001$

Tab. 3. Positive correlations between anthropometric data, blood pressure and LV paramters.

\begin{tabular}{|c|c|c|c|c|c|c|c|}
\hline & SBP & DBP & $24 \mathrm{~h} \mathrm{SBP}$ & IVS & LVM & LVEDD & LVESD \\
\hline $\mathrm{BW}$ & $* * * * \quad r<0.544$ & $* * * r<0.337$ & $* * \mathrm{r}<0.334$ & $* * * * * r<0.496$ & $* * * \mathrm{r}<0.302$ & $* * * * * \mathrm{r}<0.576$ & $* * * \mathrm{r}<0.477$ \\
\hline BMI & $* * * * \quad r<0.441$ & $* \mathrm{r}<0.301$ & $* \mathrm{r}<0.334$ & $* * * \mathrm{r}<0.351$ & $* * * * * \quad \mathrm{r}<0.436$ & $* * * * \mathrm{r}<0.399$ & $* * * \mathrm{r}<0.361$ \\
\hline & RWTh & LVEDV & LVESV & SV & LAD & & \\
\hline BW & $* * * * r<0.465$ & $* * * * * r<0.611$ & $* * * * r<0.492$ & $* * * * * \mathrm{r}<0.530$ & $* * * * * \mathrm{r}<0.472$ & & \\
\hline BMI & $* * * \mathrm{r}<0.370$ & $* r<0.372$ & & $* * * * r<0.374$ & $* * * * r<0.360$ & & \\
\hline
\end{tabular}

BW - body weight, BMI - body mass index, SBP - systolic blood pressure, DBP - diastolic blood pressure, 24h SBP - average 24 hours systolic blood pressure, IVS - interventricular septum thickness, LVM - left ventricular mass, LVEDD - left ventricular diastolic diameter, LVESD - left ventricular systolic diameter, RWTh - relative wall thickness, LVEDV - left ventricular end-diastolic volume, LVESV - left ventricular end-systolic volume, SV - stroke volume, LAD - left atrium diameter

${ }^{*} \mathrm{p}<0.01,{ }^{* *} \mathrm{p}<0.05, * * * \mathrm{p}<0.005, * * * * \mathrm{p}<0.001, * * * * * \mathrm{p}<0.0001$

tion fraction, which was not changed compared to controls. There was no systolic dysfunction in any child. Significant differencies have been also in parameters of left ventricular diastolic function. Left atrial diameter and late diastolic filling peak flow velocity (A-wave) were significantly increased. E/A ratio was significantly lower. Isovolumetric relaxation time and early diastolic filling deceleration time were higher than in controls, but without statistical significance. In children with obesity both, IVRT and DT, reached statistical significance (Tab. 2).

There were significant positive correlations between body mass, BMI and specific left ventricular parameters (Tab. 3).

\section{Discussion}

Recently, extreme increase of obesity has been reported not only in adults, but also in children (12). Obesity, predominantly in the upper body (android, visceral type) is more frequent, than obesity of the lower body (gynoid, peripheral type). Android obesity represents a strong and independent predictor of many consequences, such as dislipidemia, insulin resistance, non-alcoholic fatty liver disease and increase in mortality $(13,14)$. The waist circumference, as an indicator of intraabdominal fat mass, correlates with cardiovascular risks and aterogenous lipid profile even more significantly than BMI (15).

Many studies have proved the relation between obesity and heart failure (1). Impairment of myocardial function correlates with BMI and obesity duration (3). Recently more and more attention is paid to the so called "cardiomyopathy in obese individuals or cardiomyopathy related to obesity", which represents a risk factor of heart failure $(16,17,18,19)$. In obesity, the chronic volume overload (increased preload) leads to LV dilatation, increase in the ventricular wall tension and finally eccentric compensational LV hypertrophy (20). Several studies have showed the presence of diastolic dysfunction, related to this form of cardiomyopathy without a simultaneous occurrence of systolic dysfunction (3,21). Early identification of these abnormalities is of the upmost importance, because effective treatment can reverse these processes in early stages of diseases.

In our study, children and adolescents with obesity and overweight had significant increase in IVST, LVEDD, LVESD as well as left ventricular PWTh with positive correlation with body mass and BMI. These changes seemed to be stronger in children with more severe obesity, but the number of these children was too small for the independent statistical evaluation. Also Dorbala et al (22), pointed out the positive correlation between BMI and the LV size. The LV dilatation was present even in the case of a mild form of obesity. In our study we have confirmed the correlation between all systolic pressures and IVS, PWTh but also LVEDD. The effect of BP (a higher BP or already developed arterial hypertension) may participate in these structural myocardial changes. Similarly to the study of Di Bello et al. (16), in adult patients, in our sample of children patients with obesity and overweight, the heart volume values were significantly higher. Adult obese patients participating in the above mentioned study showed a higher LVEDV resulting from the increased LV preload. Based on the Frank-Starling mechanism, a significant increase of the stroke volume and cardiac output in 
obese individuals occur, compared to individuals with normal body mass. A direct consequence of this phenomenon, observed also in our obese children, is the significant enlargement of the left atrium (LA) in obese individuals. Moreover, we have confirmed also a positive correlation between body mass and LV systolic function parameters. There are several factors that cause LV dilatation due to the increased BMI, including hyperdynamic volume overload (increased blood volume and capillary flow), neurohumoral activation and increase of oxidative stress $(1,23,24)$. Based on the results of our study, the global systolic function (LV ejection fraction) did not vary in children with overweight, obesity and normal body mass and the LV systolic dysfunction was not identified in any of our patients, similarly to the study of Dorbala et al (22), where there were no changes even in the presence of severe obesity. There are also many other studies focusing mainly on adult patients, which do not confirm systolic dysfunction in obese patients [3]. Some authors e.g. Iacobellis et al. (4), reported changes of the LV systolic function in addition to structural changes. As the results suggest, even in children the obesity is related to certain structural heart changes and LV remodelling. However, despite the global LV systolic function still being preserved, altered parameters of the diastolic function and dilated left atrium can be observed, and the changes tend to be more significant with the increase of the severity of obesity. Pascual et al (3), were among the first to report cases of early myocardial damage in isolated obesity, especially when using a conventional Doppler echocardiographic examination of transmitral flow and shoved a subclinical diastolic dysfunction of LV in spite of the fact that LV systolic dysfunction has not been identified in any patient. Harada et al (25), confirmed the reduction of LV early diastolic filling in a sample of 21 asymptomatic obese children. The increased IVRT and DT in obese individuals were reported also by other authors $(16,26,27,28)$. Several other studies, evaluating the diastolic function in obese children and adolescents have been published, which correspond to our results $(25,29)$. Changes in LV diastolic function indexes occur in obese children and adolescents. The left atrium (LA) diameter in obese and overweight children in our study was significantly higher compared to children with normal body mass, similarly to the results of Iacobellis et al study (4). The study confirmed not only the correlation between LA, body mass and BMI, but also with LV size and thickness. All these results are in accordance with the findings of Yu et al (30), who came to similar conclusions in the sample of 22 obese children. However, so far it is not clear, whether the increased LA diameter in obese children represents an independent risk factor of cardiovascular diseases as it is in adults. Benjamin et al (31), confirmed that the LA dilatation is associated with an increased risk of cerebral vascular disease in males and with death in both genders. Average BP values as well as all BP values in ABPM were higher in obese and overweight children. Similar conclusions were reported also by Alpert et al (20), in their study of hypertensive children and Daniels et al (32), in a sample of 112 hypertensive children with average age of 14.2 years and in addition to the effect of body mass and BMI they also confirmed the effect of systolic BP on the LA diameter.

Overweight in children results in increased LV mass compared with the expected LV mass in normal population (33).
Body mass demonstrates a strong, positive and independent effect on the LV mass (51). Our study has confirmed a positive, statistically significant correlation between LVM and LV mass/ height index ${ }^{2.7}$ and body mass, height and BMI. Dekkers et al (34), demonstrated, that obesity is a positive predictor of LVM. In obese and overweight children included in our study, LV mass and LV mass/height index ${ }^{2.7}$ were significantly higher than in children with normal body mass. Some studies prove that obesity in children results in increased LVM in adulthood, and moreover, it precedes the occurrence of other risk factors of the metabolic syndrome $(35,36,37)$. However, the reason of the diastolic dysfunction in obesity is not quite clear. Some authors suggest, that the damage to the LV diastolic function may occur due to the insulin resistance and hyperinsulinaemia or fasting hyperglycaemia $(38,39)$. There are several factors contributing to myocardial damage. The increased stroke volume and cardiac output result in dilatation of heart compartments with the LV eccentric hypertrophy. Insulin resistance results also in increased LVM in obese patients (28). Our study points out the fact that the described changes of the LV function in children and adolescents with obesity and overweight were probably caused mainly by obesity, because neither arterial hypertension nor severe dislipidemia has been identified in any of them. All the described changes were more significant in children with severe obesity, which can imply that the described changes depend on the obesity level and they are more severe in children with a higher BMI percentile and more severe obesity.

\section{References}

1. Kenchaiah S, Evans JC, Levy D, Wilson PW, Benjamin EJ, Larson MG, Kannel WB et al. Obesity and the risk of heart failure. New Engl J Med 2002; 347: 305-313.

2. Saligova J, Schusterova I, Potocnakova L. Disorders of lipid metabolism and changes of the left ventricle in children. J Inherit Metab Dis 2006; 29 (Suppl 1): 148.

3. Pascual M, Pascual DA, Soria F, Vicente T, Hernandez AM, Tebar FJ, Valdes M. Effects of isolated obesity on systolic and diastolic left ventricular function. Heart 2003; 89: 1152-1156.

4. Iacobellis G, Ribaudo MC, Leto G, Zappaterreno A, Vecci E, Di Mario U. Influence of Excess Fat on Cardiac Morphology and Function: Study in Uncomplicated Obesity. Obes Res 2002; 10: 767-773.

5. National Institutes of Health Consensus Development Panel on the Health Implications of Obesity: health implication of obesity. Ann Intern Med 1985; 103 (Suppl 6): 1073-1077.

6. National Heart, Lung and Blood Institute: Update on the 1987 Task Force on High Blood Pressure in Children and Adolescents: A Working Group from the National High Blood Pressure Education Program. Pediatrics 1996; 98: 649-658.

7. Sahn DJ, Demaria A, Kisslo J, Weyman A. Recommendations regarding quantitation in M-mode echocardiographic: results of a survey of echocardiographic measurements. Circulation 1978; 58: 1072-1083.

8. Devereux RB, Alonso DR, Lutas EM, Gottlieb GJ, Campo E, Sachs I. Echocardiography assessment of left ventricular hypertrophy: comparison to necropsy findings. Am J Cardiol 1986; 57: 450-458. 
9. Daniels SR, Meyer RA, Liang Y, Bove KE. Echocardiographically determined left ventricular mass index in normal children, adolescents and young adults. J Am Coll Cardiol 1988; 12: 703-708.

10. De Simone G, Daniels SR, Devereux RB, Meyer RA, Roman MJ, Dedivitiis $\mathbf{O}$ et al. Left ventricular mass and body size in normotensive children and adults: assessment of allometric relations and impact of overweight. J Am Coll Cardiol 1992; 20: 1251-1260.

11. Schiller NB, Shah PM, Crawford M, Demaria A, Devereux RB, Feigenbaum H. American Society of Echocardiography Committee on Standards Subcommittee on Quantitation of Two-Dimensional Echocardiograms. Recommendations for quantitation of the left ventricle by two-dimensional echocardiography. J Am Soc Echocardiogr 1989; 41: 126-131.

12. Lobstein T, Baur LA. Policies to prevent childhood obesity in the European Union. Eur J of Public Health 2005; 15: 576-579.

13. Nguyen-Duy TB, Nichaman MZ, Church TS, Blair SN, Ross R. Visceral fat and liver fat are independent predictors of metabolic risk factors in men. Am J Physiol Endocrinol Metab 2003; 284: E1065-1071.

14. Ross R, Aru J, Freeman J, Hudson R, Jansen I. Abdominal adiposity and insulin resistance in obese men. Am J Physiol Endocrinol Metab 2002; 282: E657-663.

15. Kuk JL, Katzmarzyk PT, Nichaman MZ, Church TS, Blair SN, Ross R. Visceral fat is an independent predictor of all-cause mortality in men. Obes Res 2006; 14: 336-341.

16. Di Bello V, Santini F, Di Cori A, Pucci A, Palagi C, Delle Donne MG. Obesity cardiomyopathy: Is it reality? An ultrasonic tissue characterization study. J Am Soc Echocardiogr 2006; 19: 1063-1071.

17. Peterson LR, Waggoner AD, Schechtman KB, Meyer T, Gropler RJ, Barzilai B. Alteration in left ventricular structure and function in young healthy obese women. J Am Coll Cardiol 2004; 43: 1399-1404.

18. Willens HJ, Chakko SC, Byers P, Chirinos JA, Labrador E, Castrillon JC. Effects of weight loss after gastric bypass on right and left ventricular function assessed by tissue Doppler imaging. Am J Cardiol 2005; 95:1521-1524.

19. Rutter MK, Parise H, Benjamin EJ. Impact of glucose intolerance and insulin resistance on cardiac structure and function: sex-related differences in the Framingham heart study. Circulation 2003; 107: 448-454.

20. Alpert MA. Obesity cardiomyopathy: pathophysiology and evolution of the clinical syndrome. Am J Med Sci 2001; 321: 225-236.

21. Zarich SW, Kowalchuk GJ, Mcguire MP. Left ventricular filling abnormalities in asymptomatic morbid obesity. Am J Cardiol 1991; 68: 377-381.

22. Dorbala S, Crugnate S, Yang D, Di Carli MF. Effect of Body Mass Index on Left Ventricular Cavity Size and Ejection Fraction. Am J Cardiol 2006; 97: 725-729.

23. Engeli S, Sharma AM. The renin-angiotensin system and natriuretic peptides in obesity-associated hypertension. J Mol Med 2001; 79: 21-29.

24. Herrera MF, Deitel M. Cardiac function in massively obese patients and the effect of weight loss. Can J Surg 1991; 34: 431-434.
25. Harada K, Orino T, Takada G. Body mass index can predict left ventricular diastolic filling in asymptomatic obese children. Ped Cardiol 2001; 22: 273-278.

26. Talini E, Di Bello V, Bianchi C, Palagi C, Delle Donne MG, Penno G. Early impairment of left ventricular function in hypercholesterolemia and its reversibility after short term treatment with rosuvastatin, A preliminary echocardiographic study. Atherosclerosis 2008; 197: 346-354.

27. Sharpe JA, Naylor LH, Jones TW, Davis EA, O'Driscoll G, Ramsay J. Impact of Obesity on Diastolic Function in Subjects $\leq 16$ Years of Age. Am j Cardiol 2006; 98: 691-693.

28. Wong CHY, O'Moore-Sullivan T, Leano R, Byrne N, Beller E, Marwick TH. Alterations of Left Ventricular Myocardial Characteristics Associated With Obesity. Circulation 2004; 110: 3081-3087.

29. Powell BD, Redfield MM, Bybee KA, Freeman WK, Rihal CHS. Association of Obesity With Left Ventricular Remodeling and Diastolic Dysfunction in Patients Without Coronary Artery Disease. Am J Cardiol 2006; 98: 116-120.

30. Jin Yu J, Hyun Yeom H, Chung S, Park Y, Hwan Lee D. Left Atrial Diameters in Overweight Children with Normal Blood Pressure. J Pediatr 2006; 148: 321-325.

31. Benjamin EJ, D'Agostino RB, Belanger AJ, Wolf PA, Levy D. Left atrial size and the risk of stroke and death: the Framingham Heart Study. Circulation 1995; 92: 835-841.

32. Daniels SR, Witt SA, Glascock BG, Khoury PR, Kimball TR. Left atrial size in children with hypertension: the influence of obesity, blood pressure, and left ventricular mass. J Pediatr 2002; 141: 186-190.

33. Daniels SR, Loggie JMH, Khoury P, Kimball TR. Left ventricular geometry and severe left ventricular hypertrophy in children and adolescents with essential hypertension. Circulation 1998; 97: 1907-1911.

34. Dekkers C, Treibeer SF, Kapuku G, van den Oord EJCG, Sneider H. Growth of left ventricular mass in African American and European American youth. Hypertension 2002; 39: 943-951.

35. Frommelt PC. Echocardiographic measures of diastolic function in pediatric heart disease. Curr Opin Cardiol 2006; 21: 194-199.

36. Srinivasan SR, Myers L, Berenson GS. Predictability of childhood adiposity and insulin for developing insulin resistance syndrome (syndrome X) in young adulthood: the Bogalusa Heart Study. Diabetes 2002; 51: 204-209.

37. Li X, Li S, Ulusoy E, Chen W, Srinivasan SR, Berenson GS. Childhood adiposity as a predictor of cardiac mass in adulthood: the Bogalusa Heart Study. Circulation 2004; 119: 3488-3492.

38. Mureddu GF, De Simone G, Greco R, Rosato GF, Contaldo F. Left ventricular filling pattern in uncomplicated obesity. Am J Cardiol 1996; 77: 509-514.

39. Jain A, Avendano G, Dharamsey S. Left ventricular diastolic function in hypertension and role of plasma glucose and insulin: comparison with diabetic heart. Circulation 1996; 93: 1396-1402.

Received August 15, 2011. Accepted November 30, 2011. 